\title{
Don't Take It 'Lytely': A Case of Acute Tetany
}

\author{
McKenna M. Johnson ${ }^{1}$, Satya Patel ${ }^{2}$, Jason Williams ${ }^{3}$ \\ 1. Internal Medicine, David Geffen School of Medicine at University of California Los Angeles, Los Angeles, USA 2. \\ Internal Medicine, University of California Los Angeles, Los Angeles, USA 3. Internal Medicine, Louis Stokes Cleveland \\ Veterans Affairs Medical Center, Cleveland, USA
}

Corresponding author: McKenna M. Johnson, mckennajohnson@mednet.ucla.edu

\begin{abstract}
The most common causes of tetany are hypocalcemia, hypomagnesemia, hypokalemia, and alkalosis. Most case reports of tetany in the literature include some combination of the above metabolic derangements leading to non-life-threatening symptoms. We present a unique case of severe life-threatening tetany in a 38-year-old female with a history of Crohn's disease. She was previously dependent on total parenteral nutrition (TPN) but discontinued TPN two weeks prior to presentation due to the improvement of her Crohn's symptoms with a new medication regimen. We propose that malabsorption led to multiple electrolyte abnormalities, resulting in acute tetany that subsequently caused rhabdomyolysis. This case reviews the most common causes of acute tetany and highlights the interaction between electrolytes implicated in both tetany and rhabdomyolysis. It also emphasizes the importance of considering tetany as a diagnosis in a patient with unstable vital signs and diffuse muscle spasms.
\end{abstract}

Categories: Endocrinology/Diabetes/Metabolism, Internal Medicine

Keywords: tetany, hypocalcemia, respiratory alkalosis, crohns disease, hypokalemia, rhabdomyolysis, hypomagnesemia

\section{Introduction}

Tetany is a condition in which an abnormal serum electrolyte concentration, such as hypocalcemia, hypomagnesemia, hypokalemia, or alkalosis, leads to neuromuscular irritability. Often, the etiology is not due to a single cause but rather a combination of electrolyte derangements. Several cases in the literature describe the development of tetany in young females with malabsorption due to inflammatory bowel disease, celiac disease, or bulimia, who are found to have multiple electrolyte abnormalities [1-3]. The majority of published cases of tetany describe non-life-threatening symptoms. We present a case of acute life-threatening tetany in a 38-year-old female with chronic malabsorption due to Crohn's disease.

Received 07/06/2019 Review began 07/28/2019 Review ended 10/03/2019 Published 10/05/2019

() Copyright 2019 Johnson et al. This is an open access article distributed under the terms of the Creative Commons Attribution License CC-BY 3.0., which permits unrestricted use, distribution, and reproduction in any medium, provided the original author and source are credited.

\section{Case Presentation}

A 38-year-old female with a history of treatment-refractory Crohn's disease with prior ileocolectomy presented to the emergency department with acute onset of severe muscle cramping and spastic paralysis. She failed numerous therapies for Crohn's disease in the past, including mesalamine, 6-mercaptopurine, infliximab, adalimumab, and vedolizumab. As a result, she developed chronic malabsorption and intermittently required total parenteral nutrition (TPN). Her primary indication for TPN was refractory hypokalemia. However, due to a new treatment regimen with ustekinumab initiated two months prior, her symptoms improved, and she stopped TPN therapy two weeks prior to presentation. She reported a normal appetite and good oral intake during this time period. She endorsed mild diarrhea, which was consistent with her baseline after the ileocolectomy. Of note, she did not have any laboratory tests since the cessation of TPN.

A few hours before presenting to the emergency department, she was setting up tables for a work conference when she noticed sudden-onset perioral numbness and tingling of her hands. Within minutes, she developed progressive and severe muscle cramping. She described finger extension, wrist flexion, arm flexion against her chest, leg extension, rotational torticollis to the left, and shortness of breath. On arrival to the emergency department, her vital signs were as follows: temperature $40.7^{\circ} \mathrm{C}$, heart rate 192 beats/minute, blood pressure 102/60 $\mathrm{mmHg}$, respiratory rate 40 breaths/minute, and normal oxygen saturation on room air. Physical exam revealed an alert, diaphoretic female in acute distress with diffuse abdominal tenderness and diffuse muscle spasm. Her initial labs included the following: potassium $4.0 \mathrm{mEq} / \mathrm{L}$, lactate $87 \mathrm{mg} / \mathrm{dL}(9.65 \mathrm{mmol} / \mathrm{L})$, creatinine kinase (CK) $150 \mathrm{U} / \mathrm{L}$, and a venous blood gas with $\mathrm{pH}$ of 7.46 and pCO2 of $24 \mathrm{mmHg}$. Additional laboratory values can be seen in Table 1. Serum calcium and magnesium were not initially tested. Her initial electrocardiogram (EKG) can be seen in Figure 1. CT head without contrast showed no sign of intracranial hemorrhage. Due to her acute and severe presentation, she was empirically treated for sepsis with intravenous (IV) fluids, antibiotics, and antifungals. IV fluids administered included 1L normal saline and 1.5L lactated ringers. CT abdomen and pelvis with IV contrast was notable for hyperenhancement and bowel wall edema of the distal small bowel, most likely due to inflammatory bowel disease or infectious enteritis (Figure 2). Within three hours of arrival, her symptoms resolved and lactate normalized. However, her serum CK increased significantly, peaking at 


\section{Cureus}

12,380 U/L. Six hours after presentation, her labs were notable for the following: potassium $2.4 \mathrm{mEq} / \mathrm{L}$, calcium $6.3 \mathrm{mg} / \mathrm{dL}$ (corrected calcium $7.8 \mathrm{mg} / \mathrm{dL}$ ), and magnesium $0.9 \mathrm{mg} / \mathrm{dL}$. Aggressive electrolyte repletion was initiated. Her clinical exam normalized the following morning, and IV antibiotics and antifungals were discontinued. Gastroenterology was consulted to address her severe malabsorption and recommended an additional dose of ustekinumab along with the resumption of TPN.

\begin{tabular}{|c|c|c|c|c|c|c|c|c|c|}
\hline \multirow[t]{2}{*}{ Laboratory Values } & \multicolumn{2}{|l|}{ Day 0} & \multicolumn{6}{|l|}{ Day 1} & \multirow{2}{*}{$\begin{array}{l}\text { Day } 2 \\
\text { 5:00 PM }\end{array}$} \\
\hline & $\begin{array}{l}9: 30 \\
\text { PM }\end{array}$ & $\begin{array}{l}12: 00 \\
\text { AM }\end{array}$ & $\begin{array}{l}12: 45 \\
\text { AM }\end{array}$ & $\begin{array}{l}4: 00 \\
\mathrm{AM}\end{array}$ & $\begin{array}{l}8: 00 \\
\text { AM }\end{array}$ & $\begin{array}{l}10: 00 \\
\text { AM }\end{array}$ & $\begin{array}{l}\text { 6:00 } \\
\text { PM }\end{array}$ & 10:00 PM & \\
\hline Sodium (mmol/L) & 140 & & & 144 & & 143 & 146 & & 145 \\
\hline Potassium (mmol/L) & 4 & & & 2.4 & & 3 & 3.1 & & 3.2 \\
\hline Chloride (mmol/L) & 100 & & & 105 & & 107 & 110 & & 109 \\
\hline Bicarbonate (mmol/L) & 18 & & & 23 & & 22 & 19 & & 20 \\
\hline BUN (mg/dL) & 8 & & & 7 & & 14 & 4 & & 16 \\
\hline Creatinine (mg/dL) & 0.8 & & & 0.86 & & 0.76 & 0.7 & & 0.63 \\
\hline Lactate (mg/dL) & 87 & 13 & 9 & & & & & & \\
\hline Creatinine Kinase (U/L) & 150 & & 5077 & 9066 & & 12380 & 12205 & & 8555 \\
\hline Calcium (mg/dL) & & & & 6.3 & & 7.5 & 7.4 & & 7 \\
\hline Magnesium (mEq/L) & & & & 0.9 & & 2.3 & & & 2.7 \\
\hline Phosphorous (mg/dL) & & & & & & & 1.5 & & 2.9 \\
\hline Albumin (g/dL) & & & & 2.1 & & & 2.4 & & \\
\hline $\begin{array}{l}\text { lonized Calcium, Corrected } \\
\text { (mmol/L) }\end{array}$ & & & & & & & 0.97 & & \\
\hline PTH, intact (pg/ml) & & & & & & & 32 & & \\
\hline Vitamin D, 25-OH (ng/mL) & & & & & & & 16 & & \\
\hline Venous Blood Gas (pH/pCO2) & 7.46/24 & & & & & & & & \\
\hline
\end{tabular}

\section{TABLE 1: Laboratory values during the first two days of the hospital course}

Normal range: calcium, 8.6-10.3 mg/dL; magnesium, 1.4-1.9 mEq/L; potassium, 3.6-5.3 mmol/L; phosphorous, 2.3-4.4 mg/dL

Abbreviations: BUN, blood urea nitrogen; PTH, parathyroid hormone

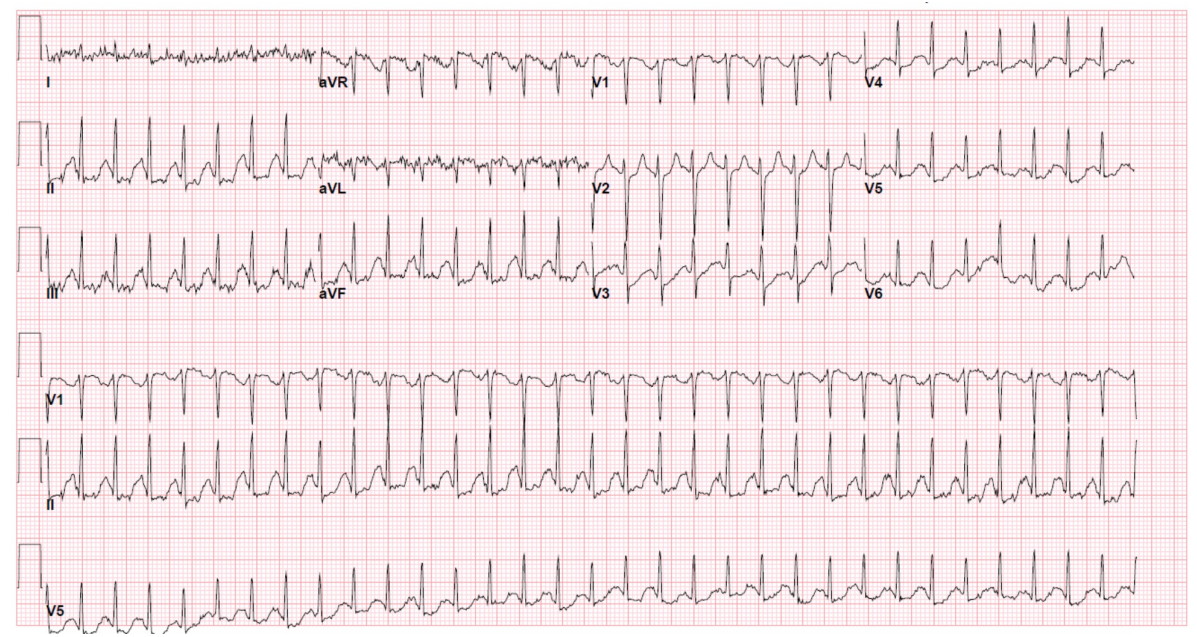




\section{Cureus}

\section{FIGURE 1: EKG during initial presentation in emergency department}

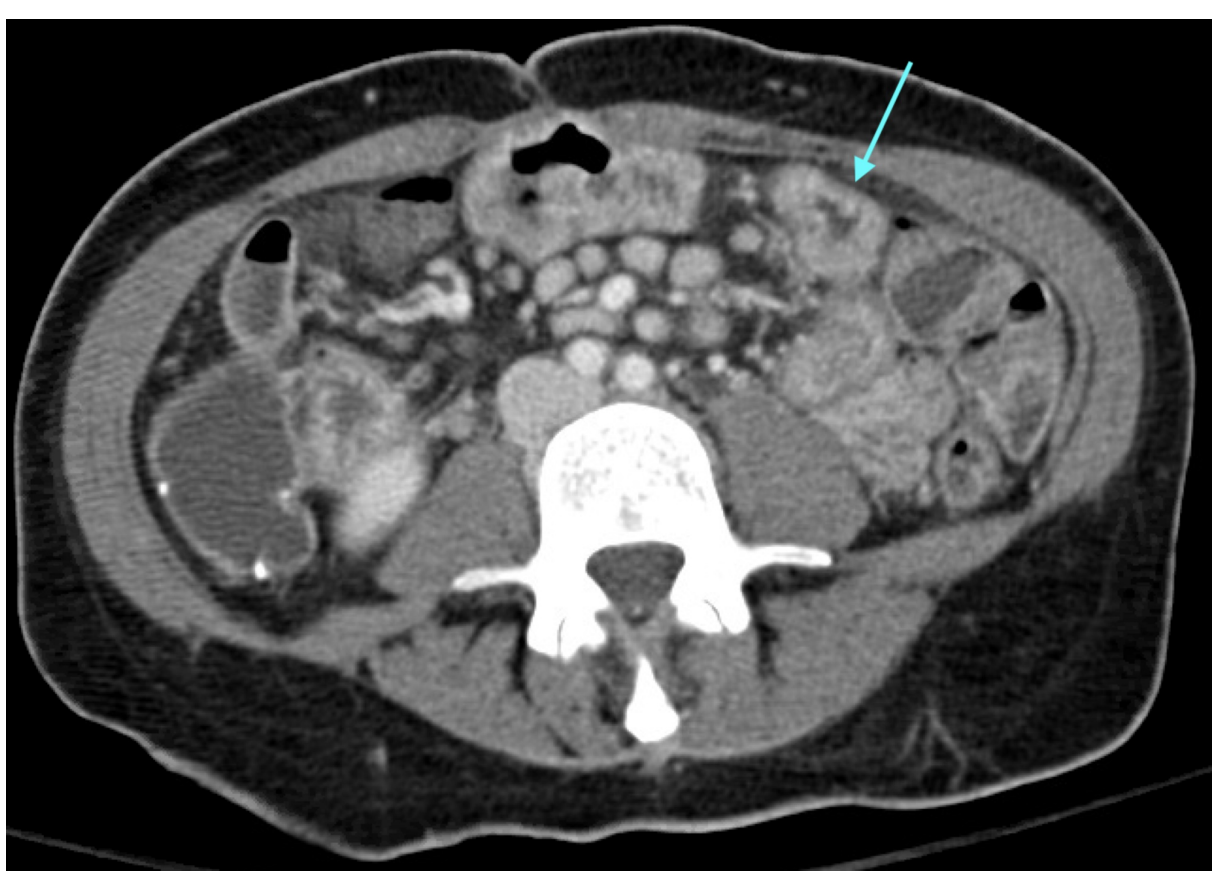

FIGURE 2: CT abdomen and pelvis with IV contrast

The blue arrow points to the region of small bowel wall edema.

\section{Discussion}

Due to the acuity of her presentation and the combination of clinical symptoms, the differential diagnosis included sepsis, seizure, malignant hyperthermia, and serotonin syndrome. However, none of these diagnoses could entirely account for her presentation. Although sepsis was considered due to her fever, tachycardia, and prior peripherally inserted central catheter (PICC), she had no obvious source, and finalized cultures revealed no evidence of infection. Seizure was considered due to her widespread muscle contractions and unusual posturing, yet her mental status was unaffected, and she had no prior history of seizures. Her hyperthermia raised suspicion for malignant hyperthermia or serotonin syndrome, but she had no exposure to neuroleptic agents or serotoninergic medications. We believe that her symptoms can be explained by acute tetany followed by acute rhabdomyolysis. Tetany likely caused her diffuse muscle spasms, leading to the development of rhabdomyolysis, which caused fevers and tachycardia. Additionally, the marked level of tachycardia at $192 \mathrm{bpm}$ was most likely due to the development of arrhythmia related to electrolyte abnormalities.

Tetany is a condition caused by peripheral nerve irritability, which leads to repetitive, high-frequency discharges. The most common signs are perioral numbness, acral paresthesias, and muscle spasms. Chovstek and Trousseau signs are two classic physical exam signs which demonstrate nerve hyperreactivity and suggest tetany. Chovstek sign is elicited by tapping the facial nerve anterior to the ear, which causes contraction of ipsilateral facial muscles, such as twitching of the lip. Trousseau sign is the development of carpopedal spasm with inflation of blood pressure cuff above systolic blood pressure for three minutes [4]. Carpopedal spasm is a specific form of involuntary cramping in the hands and feet nearly synonymous with tetany. In the hands, patients develop involuntary finger extension, wrist flexion, thumb abduction, and metacarpophalangeal joint flexion. A similar pattern of cramping occurs in the feet. In some cases of tetany, muscle cramps may progress to generalized muscle contractions, as seen in our patient. Additionally, autonomic effects can also be seen, such as diaphoresis, bronchospasm, and biliary colic [5]. When using physical exam signs to aid in the diagnosis of tetany, it is important to note that the Trousseau sign is reasonably sensitive and specific for hypocalcemic tetany, but Chovstek sign is neither sensitive nor specific. For example, in patients with hypocalcemic tetany, Trousseau sign is present in $94 \%$ of patients with hypocalcemia and only $1 \%$ of patients with normal calcium levels. However, Chovstek sign is present in $10 \%$ of patients with normal calcium levels and absent in about $30 \%$ of patients with hypocalcemia [6].

Hypocalcemia is the most well-known cause of tetany, defined as serum calcium level below $7.5 \mathrm{mg} / \mathrm{dL}$ [5]. It can also cause cardiac conduction disturbances, decreased myocardial contractility, seizure, and psychiatric 
manifestations [5]. In this patient, her initial EKG showed supraventricular tachycardia, which may have been caused by hypocalcemia (Figure 1). The swift reversal of her tetany and stabilization of clinical conditions were likely due to the use of calcium-containing Lactated Ringer's solution during initial resuscitation.

Respiratory alkalosis and hypokalemia, both seen in our patient, can also contribute to tetany. Respiratory alkalosis causes dissociation of bound hydrogen ions, which bind to free serum calcium, causing hypocalcemia. Alkalosis can also independently cause nerve irritability [7]. Furthermore, reports of tetany associated with hypokalemia in the absence of other metabolic derangements have also been reported, suggesting that hypokalemia can independently cause tetany $[8,9]$.

Hypomagnesemia is another important cause of tetany, as magnesium affects the metabolism of both calcium and potassium. Low magnesium levels may reduce the threshold for hypocalcemia to cause tetany. Although acute hypocalcemic tetany is not usually seen unless corrected serum calcium levels are below $7.5 \mathrm{mg} / \mathrm{dL}$ or serum ionized calcium levels are below $1.1 \mathrm{mmol} / \mathrm{L}$, the condition can be seen at higher calcium levels if serum magnesium levels are low $[5,10]$. Hypomagnesemia may have also contributed to both tetany and rhabdomyolysis, in our patient by affecting potassium metabolism. Low magnesium levels cause increased renal potassium wasting, which potentiates pre-existing hypokalemia. Until hypomagnesemia has been corrected, potassium repletion will not effectively correct hypokalemia [11,12].

In this patient, tetany caused diffuse muscle contractions, which precipitated acute rhabdomyolysis. The potential causes of rhabdomyolysis can be broken down into one of three categories: traumatic, nontraumatic exertional, or nontraumatic nonexertional [13]. Electrolyte disorders, particularly hypokalemia and hypophosphatemia, can cause nontraumatic nonexertional rhabdomyolysis [14,15]. In this patient, the development of generalized muscle contractions caused nontraumatic exertional rhabdomyolysis. In addition, due to the presence of persistent hypokalemia after discontinuing TPN, it is possible that she also developed preceding nontraumatic nonexertional rhabdomyolysis prior to presenting with tetany. If so, the release of intracellular phosphorous from damaged myocytes could have contributed to acute hypocalcemic tetany by binding free serum calcium (Figure 3) [16].

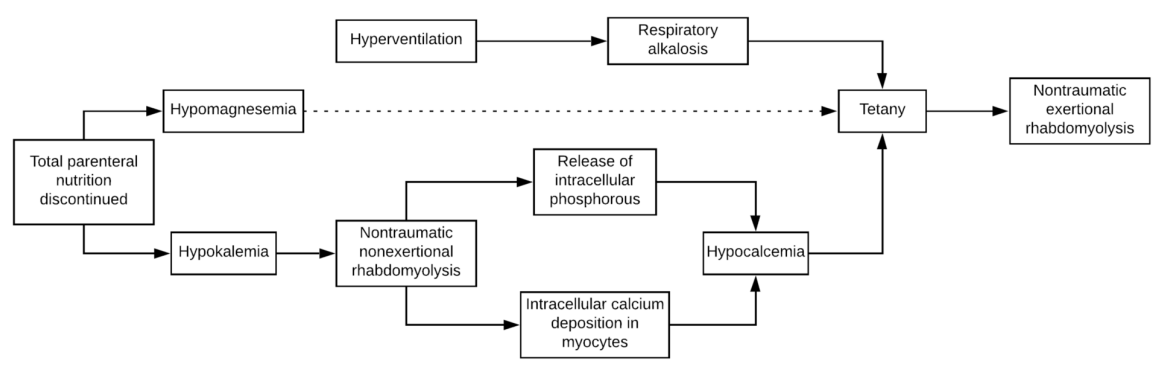

\section{FIGURE 3: Theory of clinical events if triggered by hypokalemia-induced rhabdomyolysis}

This patient's alarming clinical picture was ultimately caused by the development of multiple concurrent electrolyte derangements leading to life-threatening tetany. In the combined setting of hypocalcemia, hypomagnesemia, and respiratory alkalosis, our patient developed acute tetany with diffuse muscle contractions. The development of diffuse muscle contractions then precipitated a more severe nontraumatic exertional rhabdomyolysis.

\section{Conclusions}

In the setting of severe malabsorption, metabolic derangements can have life-threatening consequences. In this case, undetected ongoing malabsorption of potassium, magnesium, and calcium caused a presentation of acute, severe tetany. The vignette highlights the importance of detecting and correcting electrolyte abnormalities as they can ultimately lead to hemodynamic instability.

\section{Additional Information \\ Disclosures}

Human subjects: Consent was obtained by all participants in this study. Conflicts of interest: In compliance with the ICMJE uniform disclosure form, all authors declare the following: Payment/services info: All authors have declared that no financial support was received from any organization for the submitted work. Financial relationships: All authors have declared that they have no financial relationships at present or within the previous three years with any organizations that might have an 
interest in the submitted work. Other relationships: All authors have declared that there are no other relationships or activities that could appear to have influenced the submitted work.

\section{References}

1. Nomoto H, Miyoshi H, Nakamura A, Nagai S, Kitao N, Shimizu C, Atsumi T: A case of osteomalacia due to deranged mineral balance caused by saccharated ferric oxide and short-bowel syndrome: A case report. Medicine (Baltimore). 2017, 96:e8147. Accessed: July 24, 2019: https://www.ncbi.nlm.nih.gov/pmc/articles/PMC5626297/. 10.1097/md.0000000000008147

2. Posul E, Can G, Tekelioglu V, et al.: Tetany with hypomagnesemia, hypokalemia, and hypocalcemia as initial presentation of celiac disease. Gastroenterol Nurs. 2018, 41:66-67. 10.1097/SGA.0000000000000172

3. Tachamo N, Timilsina B, Lohani S, Nazir S: Spontaneous bilateral carpopedal spasm in a bulimic patient . BMJ Case Rep. 2017, 2017:Accessed: July 24, 2019: 10.1136/bcr-2017-222726

4. Cooper MS, Gittoes NJL: Diagnosis and management of hypocalcaemia . BMJ. 2008, 336:1298. 10.1136/bmj.39582.589433.BE

5. Tohme JF, Bilezikian JP: Hypocalcemic emergencies. Endocrinol Metab Clin North Am. 1993, 22:363-375. 10.1016/S0889-8529(18)30171-3

6. Jesus JE, Landry A: Chvostek's and Trousseau's Signs. N Engl J Med. 2012, $367: e 15$. 10.1056/nejmicm1110569

7. Moon HS, Lee SK, Chung JH, In CB: Hypocalcemia and hypokalemia due to hyperventilation syndrome in spinal anesthesia - A case report. Korean J Anesthesiol. 2011, 61:519-523. 10.4097/kjae.2011.61.6.519

8. Ault MJ, Geiderman J: Hypokalemia as a cause of tetany. West J Med. 1992, 157:65-67.

9. Jacob J, De Buono B, Buchbinder E, Rolla AR: Tetany induced by hypokalemia in the absence of alkalosis . Am J Med Sci. 1986, 291:284-285. 10.1097/00000441-198604000-00011

10. Fatemi S, Ryzen E, Flores J, Endres DB, Rude RK: Effect of experimental human magnesium depletion on parathyroid hormone secretion and 1,25-dihydroxyvitamin D metabolism. J Clin Endocrinol Metab. 1991, 73:1067-1072. 10.1210/jcem-73-5-1067

11. Huang CL, Kuo E: Mechanism of hypokalemia in magnesium deficiency . J Am Soc Nephrol. 2007, 18:26492652. 10.1681/asn.2007070792

12. Whang R, Whang DD, Ryan MP: Refractory potassium repletion. A consequence of magnesium deficiency . Arch Intern Med. 1992, 152:40-45. 10.1001/archinte.1992.00400130066006

13. Huerta-Alardín AL, Varon J, Marik PE: Bench-to-bedside review: Rhabdomyolysis - an overview for clinicians. Critical Care. 2004, 9:158. 10.1186/cc2978

14. Shintani S, Shiigai T, Tsukagoshi H: Marked hypokalemic rhabdomyolysis with myoglobinuria due to diuretic treatment. Eur Neurol. 1991, 31:396-398. 10.1159/000116702

15. Singhal PC, Kumar A, Desroches L, Gibbons N, Mattana J: Prevalence and predictors of rhabdomyolysis in patients with hypophosphatemia. Am J Med. 1992, 92:458-464. 10.1016/0002-9343(92)90740-3

16. Efstratiadis G, Voulgaridou A, Nikiforou D, Kyventidis A, Kourkouni E, Vergoulas G: Rhabdomyolysis updated. Hippokratia. 2007, 11:129-137. 